\title{
AN UPDATED SYNOPSIS OF LUDWIGIA (ONAGRACEAE) IN MALESIA
}

\author{
Received March 28, 2021; accepted April 12, 2021
}

\section{M. TURNER}

Singapore Botanical Liaison Officer, Royal Botanic Gardens Kew, UK. Singapore Botanic Gardens, National Parks Board, 1 Cluny Road, Singapore 259569, Republic of Singapore.

E-mail: I.Turner@kew.org

\begin{abstract}
TURNER, I. M. 2021. An updated synopsis of Ludwigia (Onagraceae) in Malesia. Reinwardtia 20(1): 9-16. - A synopsis of the seven native or naturalised species of Ludwigia (Onagraceae) occurring in Malesia is presented. The newly established Ludwigia leptocarpa is included. The paper incorporates a key to the species, synonymy and typification. Lectotypes are designated for 25 names, including five at the second step.
\end{abstract}

Key words: Ludwigia leptocarpa, South-East Asia, typification, weeds.

\begin{abstract}
ABSTRAK
TURNER, I. M. 2021. Pembaruan sinopsis Ludwigia (Onagraceae) di Malesia. Reinwardtia 20(1): 9-16. Tulisan ini menyajikan sinopsis tujuh jenis asli atau jenis yang bernaturalisasi dari Ludwigia (Onagraceae) yang terdapat di Malesia, termasuk di dalamnya yang baru dikukuhkan yaitu Ludwigia leptocarpa. Tulisan ini menggabungkan kunci jenis, sinonim dan tipifikasi. Ditetapkan pula lektotipe untuk 25 nama, termasuk lima pada tahapan kedua.
\end{abstract}

Kata kunci: Asia Tenggara, gulma, Ludwigia leptocarpa, tipifikasi.

\section{INTRODUCTION}

Ludwigia is a genus of 82 species distributed through the tropical, subtropical and warm temperate regions of the world; most diverse in the New World (Wagner et al., 2007). Ludwigia is sister to the rest of the Onagraceae and placed in its own subfamily: Jussiaeoideae. The genus has been divided into 23 sections (Wagner et al., 2007), but recent molecular analyses found limited support for this system of infrageneric division (Liu et al., 2017).

The revision of Ludwigia (Onagraceae) in the Old World by Raven (1964) has stood the test of time very well; and his account for Flora Malesiana (Raven, 1977) equally so. Raven (1977) recognised six species for Malesia. While this is a very small number compared with the size of the complete flora of the region, the species are quite important as weeds, particularly in ricegrowing areas (Kostermans et al., 1987). A newly established invasive species and some further clarification of typification make it worthwhile in publishing an updated synopsis of the genus for Malesia.

\section{MATERIALS AND METHODS}

The paper is largely based on study of material in the herbarium of the Royal Botanic Gardens Kew $(\mathrm{K})$. In addition, on-line information was searched for information on type holdings. Recent literature was scanned and relevant references are cited under the description and distribution heading for each species.

\section{RESULTS AND DISCUSSION}

LUDWIGIA L.

Ludwigia L., Sp. Pl. 1 (1753) 118, as Ludvigia. Type: Ludwigia alternifolia L., lectotype designated by Britton \& Brown, Ill. Fl. N. U.S. ed. 2. 2 (1913) 586.

Isnardia L., Sp. Pl. 1 (1753) 120. - Dantia Boehm. in Ludwig, Def. Gen. Pl. (1760) 388, nom. illegit. (superfl.). - Quadricosta Dulac, Fl. HautesPyrénées (1867) 329, nom. illegit. (superfl.). Type: Isnardia palustris L.

Jussiaea L., Sp. Pl. 1 (1753) 388. - Ludwigia sect. Jussiaea (L.) Baill., Hist. Pl. 6 (1877) 463. Type: Jussiaea repens L., lectotype designated by Hitchcock \& Greene, Prop. Brit. Bot. (1929) 153. Cubospermum Lour., Fl. Cochinch. (1790) 258, 275. - Type: Cubospermum palustre Lour.

Prieuria DC, Prodr. 3 (1828) 58. - Type: Prieuria senegalensis DC.

Adenola Raf., Aut. Bot. (1840) 36. - Type: Adenola grandiflora (Michx.) Raf.

Diplandra Raf., Aut. Bot. (1840) 35, nom. illegit., non Diplandra Bertero (1830), nec Diplandra Hook. \& Arn. (1838). - Type: Diplandra decurrens (Walt.) Raf., lectotype designated by Pennell, Bull. Torrey Bot. Club 48 (1921) 92.

Corynostigma C.Presl., Epim. Bot. (1851 ['1849']) 218. - Type: Corynostigma jussiaeoides C.Presl. 
Nematopyxis Miq., Fl. Ned. Ind. 1(1) (1856) 626, 630. - Type: Nematopyxis prostrata (Roxb.) Miq.

Oocarpon Micheli, Flora 57 (1874) 303. — Type: Oocarpon jussiaeoides Micheli

Ludwigia [unranked] Ludwigiantha Torr. \& A.Gray, Fl. N. Amer. 1 (1840) 526. - Ludwigiantha (Torr. \& A.Gray) Small, Bull. Torrey Bot. Club 24 (1897) 178. - Type: Ludwigia arcuata Walt.

Jussiaea sect. Fissendocarpa Haines, J. Proc. Asiat. Soc. Bengal 15 (1919) 314. - Fissendocarpa (Haines) Bennet, J. Bombay Nat. Hist. Soc. 67 (1970) 125. - Type: Jussiaea fissendocarpa Haines

\section{Key to Ludwigia species in Malesia}

1a. Sepals generally 5 , sometimes more ..........2

1 b. Sepals generally $4 \ldots \ldots \ldots \ldots \ldots \ldots \ldots \ldots \ldots . . . \ldots \ldots$

2a. Plant with creeping or floating stems; petals white with a yellow base; pedicel of capsule $2.5-5.5 \mathrm{~cm}$ long; each seed firmly embedded in a block of endocarp........1. L. adscendens

2b. Plant erect; petals entirely yellow; pedicel of capsule to $2 \mathrm{~cm}$ long; each seed surrounded by an easily detached horseshoe-shaped section of endocarp..............3. L. leptocarpa

3a. Sepals less than $5 \mathrm{~mm}$ long......................4

3b. Sepals more than $6 \mathrm{~mm}$ long....................6

4a. Capsules with 4 longitudinal rows of seeds clearly discernible when dry...7. L. prostrata

4b. Capsules more or less terete when dry, longitudinal rows of seeds not discernible .......... 5

5a. Capsules with two sorts of seed, free in apical portion, embedded in corky endocarp below ............................ 2. L. hyssopifolia 5b. Capsules with seeds all free ......5. L. perennis

6a. Bracteoles ca. $8 \mathrm{~mm}$ long, capsules obconcal..............................6. L. peruviana

6b. Bracteoles minute, $c a .0 .5 \mathrm{~mm}$ long, capsule cylindrical with 8 longitudinal ridges or ribs 4. L. octovalvis

\section{LUDWIGIA ADSCENDENS (L.) H.Hara}

Ludwigia adscendcens (L.) H.Hara, J. Jap. Bot. 28 (1953) 291. - Jussiaea adscendens L., Mant. Pl. (1767) 69. - Type: India. Mysore, Hassan District, Belur-Gendehally Road, 17 September 1969, C. Saldanha 15027 (Neotype MO [MO2334663], designated by Raven et al. in Jarvis (ed.), Order out of Chaos (2007) 605).

Jussiaea repens L., Sp. Pl. (1753) 388, non Ludwigia repens J.R.Forst. (1771). - Type: [published illustration] 'Nir-carambu' in Rheede, Hort. Malab. 2: t. 51. 1679 (Lectotype designated by Raven in Jarvis \& al. (ed.), Regnum Veg. 127 (1993) 58).
Cubospermum palustre Lour., Fl. Cochinch. (1790) 275. - Type: Loureiro s.n. (Lectotype BM [BM000793045], designated by Merrill, Trans. Am. Phil. Soc. 24 (1935) 289).

Jussiaea fluviatilis Blume, Bijdr. Fl. Ned. Ind. (1827) 1132. - Type: Java, Blume 1447 (Lectotype L [L0008654], designated by Raven, Reinwardtia 6 (1964 ['1963']) 387 at the first step and at the second step here; possible Isolectotype L0008653).

Jussiaea floribunda Griff., Not. Pl. Asiat. 4 (1854) 688. - Type: Burma, Mergui, Sept 1834, Griffith (not traced).

Jussiaea repens var. glaberrima Kuntze, Revis. Gen. Pl. 1 (1891) 251. - Type: Java, Batavia, May 1875, Kuntze 4180 (Lectotype NY [NY00232243], designated here).

Jussiaea repens var. pilosa Kuntze, Revis. Gen. Pl. 1 (1891) 251. - Type: Cambodia, March 1875, Kuntze s.n. (Lectotype NY [NY00232245], designated here).

Jussiaea repens forma albiflora Hochr., Candollea 2 (1925) 479. - Type: Java, Preanger, étangs tièdes de Tjipanas près Garroet, 26 October 1904, Hochreutiner 2208 (Lectotype G [G00013806], designated by Raven, Reinwardtia 6 (1964 ['1963’]) 388).

Description and distribution. Raven (1964: 387), Vu \& Vidal (1973: 26), Khan \& Shamsunnahar (1977: 3), Raven (1977: 104), Kostermans et al. (1987: 368), Thompson (1990: 227), Wagner (1995: 341), Barua (2010: 60), Boufford \& Raven (2014: 602), Ummul-Nazrah (2017: 55).

\section{LUDWIGIA HYSSOPIFOLIA (G.Don) Exell}

Ludwigia hyssopifolia (G.Don) Exell, Garcia de Orta 5 (1957) 471. - Jussiaea hyssopifolia G.Don, Gen. Hist. 2 (1832) 693. - Type: St Thomas [São Tomé], G. Don 42 (Holotype BM [BM000528814]).

Jussiaea linifolia Vahl, Eclog. Amer. 2 (1798) 32. - Fissendocarpa linifolia (Vahl) Bennet, J. Bombay Nat. Hist. Soc. 67 (1970) 126. - Ludwigia linifolia (Vahl) R.S.Rao, Fl. Goa, Diu, Dadra \& Nagarhaveli 1 (1985) 179, nom. illegit., non Poir. (1814). - Type: America meridionali, von Rohr 188 (Lectotype C [C10016131], designated by Raven, Reinwardtia 6 (1964 ['1963']) 385).

Jussiaea micrantha Kunze, Index Seminum (LZ, Lipsiensis) 1847 (1848) 2; Linnaea 24 (1851) 177. - Ludwigia micrantha (Kunze) H.Hara, J. Jap. Bot. 28 (1953) 293. - Lectotype (designated by Joncker, Fl. Suriname 3(2) (1942) 19): Surinam, Kegel 909 (GOET042401).

Jussiaea weddellii Micheli, Flora 57 (1874) 301, as Weddelii. - Type: Brazil, Weddell 2334 (Lectotype P [P01819501], designated by Raven, 
Reinwardtia 6 (1964 ['1963']) 385).

Jussiaea fissendocarpa Haines, J. Proc. Asiat. Soc. Bengal 15 (1920 ['1919']) 313. - Type: India, Bihar, Purneah, 21 December 1918, H.H. Haines 4541 (Lectotype CAL [CAL0000010226], designated here; Isolectotype K [K001129806]).

Description and distribution. Raven (1964: 385), Vu \& Vidal (1973: 24); Khan \& Shamsunnahar (1977: 4), Raven (1977: 104), Kostermans et al. (1987: 370), Thompson (1990: 227), Wagner (1995: 349), Boufford \& Raven (2014: 603), Ummul-Nazrah (2017: 56).

Notes. Raven (1964) cited the type of Jussiaea weddellii as 3331. I take this to be a typographical error for 2334.

\section{LUDWIGIA LEPTOCARPA (Nutt.) H.Hara}

Ludwigia leptocarpa (Nutt.) H.Hara, J. Jap. Bot. 28 (1953) 292. - Jussiaea leptocarpa Nutt., Gen. N. Am. Pl. 1 (1818) 279. - Type: Nuttall s.n. (Lectotype PH [PH00016622], designated by Brenan in Turrill \& Milne-Redhead, Fl. Trop. E. Africa, Onagraceae (1953) 16).

Jussiaea pilosa Kunth, Nov. Gen. Sp. 6 (1823) 101, t. 532. - Jussiaea variabilis var. pilosa (Kunth) Kuntze, Revis. Gen. P1. 1 (1891) 251. Type: Colombia, Río Apuro, Bonpland 375 (Lectotype P-Bonpl. [P00679565], designated by Brenan in Turrill \& Milne-Redhead, Fl. Trop. E. Africa, Onagraceae (1953) 16).

Jussiaea velutina G.Don, Gen. Syst. 2 (1832) 695. - Type: São Tomé, G. Don s.n. (Holotype BM [BM000957914]).

Jussiaea surinamensis Miq., Linnaea 18 (1844) 370. - Type: Surinam, Oranjewoud, langs de zandweg, October 1842, Focke 721 (Holotype U [U0005340]).

Jussiaea schottii Micheli, Flora 57 (1874) 302. Type: Brazil, Rio de Janeiro, Schott 4192 (Lectotype W, designated by Raven, Reinwardtia 6 (1964 ['1963']) 375).

Jussiaea pilosa var. robustior Donn.Sm., Bot. Gaz. 16 (1891) 6. - Type: Guatemala, Zacatepequez, April 1890, Donnell Smith 2123 (Lectotype US [US00123986], designated here; Isolectotypes GH, K [K000533292], NY).

Jussiaea pilosa var. pterocarpa Hassler, Fedde Rep. Sp. Nov. 12 (1913) 274. - Type: Paraguay, Gran Chaco, Santa Eliza, December 1903, Hassler 2708 (Lectotype B [B 10 0367937], designated here; isolectotypes BM, K, MPU, NY, POM).

Jussiaea seminuda H.Perr., Not. Syst. 13 (1947) 146. - Type: Madagascar, Marais de Mahabo, June 1926, H. Perrier de la Bâthie 17641 (Lectotype P [P00412973], designated at the first step by Raven, Reinwardtia 6 (1964 ['1963’]) 376 and at the second step here; Isolectotypes $\mathrm{K}$ [K000310868], P [P00412974, P00412975]).

Description and distribution. Raven (1964: 375).

Notes. The first report of this species being found in Malesia came from Papua New Guinea. Tucker (2017: 96) found that the species is invading riparian areas, wetlands and swamps in Papua New Guinea. I have also seen a specimen (FRI 84523) collected from Gunong Ledang, Johore, Peninsular Malaysia. This species should certainly looked out for in other parts of the Malesian region.

4. LUDWIGIA OCTOVALVIS (Jacq.) P.H.Raven Ludwigia octovalvis (Jacq.) P.H.Raven, Kew Bull. 15 (1962 ['1961']) 476. - Oenothera octovalvis Jacq., Enum. Syst. Pl. (1760) 19. - Jussiaea octovalvis (Jacq.) Sw., Observ. Bot. (1791) 142. Jussiaea octofila DC., Prodr. 3 (1828) 57, nom. illegit. (superfl.). - Jussiaea suffruticosa var. octofila H.Lev., Le Monde des Plantes (1894) 3. Ludwigia octovalvis var. octofila Alain, Bull. Torrey Bot. Club 90 (1963) 191, nom. illegit. (superfl.). - Type: [published illustration] Plumier, Pl. Am. (7) (1758) t. 175, f. 1 (Lectotype designated by Bizzarri \& Raven, Webbia 27 (1972) 490).

Jussiaea pubescens L., Sp. Pl. ed. 2 (1762) 555.Ludwigia pubescens (L.) H.Hara, J. Jap. Bot. 28 (1953) 293. - Type: Anon. s.n. (Lectotype LINN 552.3, designated by Dorr \& Wiersema, Taxon 59 (2010) 1573).

Jussiaea hirsuta Mill., Gard. Dict. ed. 8 (1768) no. 5. - Type: Houston s.n. (Lectotype BM [BM000957916], designated by Raven, Reinwardtia 6 (1964 ['1963’]) 357).

Jussiaea angustifolia Lam., Encycl. 3 (1789) 331. - Jussiaea suffruticosa var. angustifolia (Lam.) F.M.Bailey, Syn. Queensl. Fl. (1883) 197. Jussiaea suffruticosa forma angustifolia (Lam.) Alston, Handb. Fl. Ceylon 6 (Suppl.) (1931) 131. - Type: Java, Anon. s.n. (Lectotype P-LA - IDC 6207 LM-243/5, designated by Raven, Reinwardtia 6 (1964 ['1963’]) 357).

Jussiaea hirta Lam., Encycl. 3 (1789) 331. Type: Brazil, Commerson s.n. (Lectotype P-LA LM-243/9, designated by Munz, Darwiniana 4 (1942) 238).

Jussiaea octonervia Lam., Encycl. 3 (1789) 332. Jussiaea suffruticosa var. octonervia (Lam.) Bertoni, Descr. Fis. Econ. Paraguay (1910) 17. Jussiaea suffruticosa subsp. octonervia (Lam.) Hassl., Bull. Soc. Bot. Genève ser. 2, 5 (1913) 271. - Type: Martinique, Anon. $33 b$ (Lectotype P-LA LM-243/3, designated by Munz, Darwini- 
ana 4 (1942) 242).

Jussiaea villosa Lam., Encycl. 3 (1789) 331. Jussiaea suffruticosa var. villosa (Lam.) F.M.Bailey, Syn. Queensl. F1. (1883) 197. Jussiaea suffruticosa forma villosa (Lam.) Alston, Handb. Fl. Ceylon 6 (Suppl.) (1931) 131. - Ludwigia pubescens var. villosa (Lam.) H.Hara, J. Jap. Bot. 28 (1953) 293. - Type: Anon. s.n., India (Lectotype P-LA LM-243/6 designated by Raven, Reinwardtia 6 (1964 ['1963']) 362).

Epilobium fruticosum Lour., F1. Cochinch. (1790) 226. - Jussiaea fruticosa (Lour.) DC., Prodr. 3 (1828) 57. - Type: not traced.

Epilobium tetragonum Lour., Fl. Cochinch. (1790) 225, nom. illegit., non L. (1753). - Jussiaea tetragona Spreng., Syst. Veg. 2 (1825) 231. Type: not traced.

Jussiaea scabra Willd., Enum. P1. 1 (1809) 449. - Type: Germany, Hort. Bot. Berol., Anon. s.n. (Lectotype B-W [B -W 08139 -01 0], designated by Raven, Reinwardtia 6 (1964 ['1963']) 362).

Jussiaea exaltata Roxb. ex Andrews, Bot. Repos. 10 (1811) t. 621, as 'Jussieua'. - Jussiaea erecta var. exaltata (Roxb. ex Andrews) Ridl., J. Bot. 59 (1921) 259. - Type: [published illustration] Andrews, Bot. Repos. 10 (1811) t. 621 (lectotype designated here).

Jussiaea exaltata Roxb., Hort. Bengal. (1814) 33, as 'Jussieua', nom. illegit., non Roxb. ex Andrews (1811). - Type: [published illustration] 'Cattucarambu', Rheede, Hort. Malab. 2 (1679) t. 50 (lectotype designated by Turner, Taxon 62 (2013) 158).

Jussiaea ligustrifolia Kunth, Nov. Gen. Sp. 6 (1823) 100. - Jussiaea suffruticosa var. ligustrifolia (Kunth) Griseb., Mem. Amer. Acad. Arts (n. s.) 8 (1860) 187. - Ludwigia pubescens var. ligustrifolia (Kunth) H.Hara, J. Jap. Bot. 28 (1953) 293. - Ludwigia octovalvis var. ligustrifolia (Kunth) Alain, Bull. Torrey Bot. Club 90 (1963) 191. - Type: Mexico, Humboldt \& Bonpland s.n. (Lectotype P-Bonpl. [P00679564], designated by Raven, Reinwardtia 6 (1964 ['1963']) 358).

Jussiaea salicifolia Kunth, Nov. Gen. Sp. 6 (1823) 99. - Type: Colombia, Guaduas, Humboldt \& Bonpland 1756 (Lectotype P-Bonpl. [P00679563], designated at the first step by Raven, Reinwardtia 6 (1964 ['1963']) 358, and at the second step here; Isolectotypes P [P00136827, P01819477]).

Jussiaea ovalifolia Sims, Bot. Mag. 52 (1824) t. 2530, as 'Jussieua'. — Type: [published illustration] Sims, Bot. Mag. 52 (1824) t. 2530 (lectotype designated here).

Jussiaea angustifolia Blume, Bijdr. Fl. Ned. Ind. (1827) 1132, nom. illegit., non Lam. (1789). Jussiaea blumeana DC., Prodr. 3 (1828) 55. Type: Java, Buitenzorg, August [sine anno], Blume 1050 (Lectotype L [L008658], designated by Raven, Reinwardtia 6 (1964 ['1963']) 358; possible Isolectotype L [L008659]).

Jussiaea burmanni DC., Prodr. 3 (1828) 57. Type: not designated.

Jussiaea marcgravii DC., Prodr. 3 (1828) 58. Type: [published illustration] Camaranbaya in Marcgravius, Historia Naturalis Brasiliae Historiae Plantarum 1 (1648) 30 (Lectotype, upper illustration on page, designated here).

Jussiaea parviflora Camb. in St. Hil., Fl. Bras. Merid. 2 (1829) 263. — Type: Brésil, Province de Minas Geraes, 1816-1821, A. de Saint Hilaire 692 (Lectotype P [P01819478], designated here, Isolectotype P [P01819479]).

Jussiaea calycina C.Presl, Reliq. Haenk. 2 (1831) 34. - Type: not traced.

Jussiaea hirsuta C.Presl, Rel. Haenk. 2 (1831) 34, nom. illegit., non Mill. (1768), nec Vell. (1829). Jussiaea haenkeana Steud., Nom. ed. 2, 1 (1840) 836. - Type: not traced.

Jussiaea macropoda C.Presl, Reliq. Haenk. 2 (1831) 35. - Jussiaea suffruticosa var. macropoda (C.Presl) Munz, Darwiniana 4 (1942) 239. - Ludwigia pubescens var. macropoda (C.Presl) H.Hara, J. Jap. Bot. 28 (1953) 293. - Ludwigia octovalvis subsp. macropoda (C.Presl) P.H.Raven, Kew Bull. 15 (1962) 476. - Ludwigia octovalvis var. macropoda (C.Presl) Shinners, Sida 1 (1964) 385. Type: In Peruvia prope urbem Lima, T.P.X. Haenke s.n. (Lectotype PRC [PRC450096], designated here).

Jussiaea venosa C.Presl, Reliq. Haenk. 2 (1831) 33. - Type: Mexico, T.P.X. Haenke s.n. (Lectotype PR, designated by Raven, Reinwardtia 6 (1964 ['1963']) 358).

Jussiaea persicariifolia Schltdl., Linnaea 12 (1838) 271, as 'persicariaefolia'. - Type: not traced.

Jussiaea persicariifolia Schltdl. forma major Schltdl., Linnaea 12 (1838) 271. — Type: Mexico, near Hacienda de la Laguna, August 1829, Schiede s.n. (Lectotype HAL [HAL0069536], designated by Raven, Reinwardtia 6 (1964 ['1963']) 358).

Jussiaea occidentalis Nutt.ex Torr. \& A.Gray., Fl. N. Am. 1 (3) (1840) 521. - Type: USA, Arkansas, Nuttall S.n. (Lectotype PH [PH00016619], designated by Raven, Reinwardtia 6 (1964 ['1963']) 358).

Jussiaea linearis Hochst., Flora 27 (1844) 425, nom. illegit., non Willd. (1799). - Jussiaea angustifolia var. linearis Harv. in Harvey \& Sond., Fl. Capensis. 2 (1862) 504. - Type: South Africa, Port Natal, Krauss 73 (Lectotype K [K000310870], designated here).

Jussiaea sagrana A.Rich. in de la Sagra, Hist. Phys. Cuba, Pl. Vasc. (1846) 534, as 'sagraeana'. Ludwigia sagrana (A.Rich.) M.Gómez, Anales Soc. Esp. Hist. Nat. 23 (1894) 66. — Type: Ramón de la Sagra s.n. (Lectotype P [P01819476], designated by Raven, Reinwardtia 6 (1964 ['1963’]) 358). 
Jussiaea velutina Kunze, Linnaea 20 (1847) 56, nom. illegit., non G.Don (1832). - Type: Austria, Beschke, cultivated (LZ $\dagger$ ).

Jussiaea costata C.Presl, Epim. Bot. (1849) 217. - Type: Philippines, Luzon, Laguna Prov., Calawang, Cuming 655 (Lectotype BM [BM000984335], designated here; isolectotypes $\mathrm{K}$, L).

Jussiaea junghuhniana Miq., Fl. Ned. Ind. 1 (1) (1856) 627. - Type: Sumatra, Padang, bij Lumut, October [sine anno], Junghuhn s.n. (Lectotype L [L0008657], designated by Raven, Reinwardtia 6 (1964 ['1963']) 363).

Jussiaea octonervia forma sessiliflora Micheli in Martius, Fl. Bras. 13 (2) (1875) 171. - Jussiaea suffruticosa var. sessiliflora (Micheli) Hassl., Bull. Soc. Bot. Genève, ser. 2,5 (1913) 271. - Ludwigia pubescens var. sessiliflora (Micheli) H.Hara, J. Jap. Bot. 28 (1953) 293. - Ludwigia octovalvis subsp. sessiliflora (Micheli) P.H.Raven, Kew Bull. 15 (1962) 476. - Ludwigia octovalvis var. sessiliflora (Micheli) Shinners, Sida 1 (1964) 385. - Type: Brazil, Rio de Janeiro, Burchell 927 (Lectotype K [K000911823], designated by Munz, Darwiniana 4 (1942) 237; Isolectotype GH [GH00054202]).

Jussiaea suffruticosa var. subglabra Thwaites ex Trimen, Handb. Fl. Ceylon 2 (1894) 233. - Type: Ceylon, Thwaites 170 (not traced).

Jussiaea suffruticosa var. angustifolia Chod. \& Hassler, Bull. Herb. Boiss. sér. 2, 3 (1903) 909, nom. illegit., non (Lam.) F.M.Bailey (1883). Type: Paraguay, Igatimi, November 1898, Hassler 5541 (Holotype G [G00442815] 2 sheets).

Jussiaea suffruticosa var. sintenisii Urb., Symb. Antill. 4 (1910) 469. - Type: Puerto Rico, prope Hato Grande in collibus graminosus ad montem "Gregorio" versus, 31 August 1885, Sintenis 2719 (Lectotype B [B 10 0367942], designated by Munz, Darwiniana 4 (1942) 242).

Jussiaea suffruticosa var. linearifolia Hassl., Repert. Spec. Nov. Regni Veg. 12 (1913) 277. - Jussiaea suffruticosa forma linearifolia (Hassl.) Munz, Darwiniana 4 (1942) 243. - Ludwigia pubescens var. linearifolia (Hassl.) A.Fern. \& R.Fern., Garcia de Orta 5 (1957) 115. - Type: Paraguay, Camp San Luis, Fiebrig 4128 (Lectotype G [G00442814], designated by Raven, Reinwardtia 6 (1964 ['1963']) 359).

Jussaia suffruticosa var. hirta Ridl., Trans. Linn. Soc. Bot. 9 (1916) 57. - Type: New Guinea, Camp VIII to IX, C. Boden Kloss s.n. (Lectotype K, designated by Raven, Reinwardtia 6 (1963) 363; Isolectotype BM [BM013717213]).

Jussiaea suffruticosa var. hawaiensis Hochr., Candollea 2 (1925) 479. - Type: Iles Sandwich [Hawaii], Kauai, Weimea-village, 21 April 1905, Hochreutiner 3592 (Lectotype G [G00442813], designated by Raven, Reinwardtia 6 (1963) 359.

Jussiaea suffruticosa var. samoensis Hochr., Can- dollea 2 (1925) 479. - Type: Hochreutiner 3280 , Samoa, île d'Upolu, lac de Lanuto, 22 March 1905 (Lectotype G [G00442809], designated by Raven, Reinwardtia 6 (1964 ['1963']) 359.

Jussiaea clavata M.E.Jones, Contr. W. Bot. 15 (1929) 131. - Type: Mexico, Acaponeta, Nayarit, 24 February 1927, M.E. Jones 22871 (Lectotype WIS [WISv0255953WIS], designated here).

Jussiaea suffruticosa auctt. plur., non L.

Description and distribution. Raven (1964: 356), $\mathrm{Vu}$ \& Vidal (1973: 20), Khan \& Shamsunnahar (1977: 6), Raven (1977: 101), Kostermans et al. (1987: 372), Thompson (1990: 226), Wagner (1995: 349), Barua (2010: 65), Boufford \& Raven (2014: 603), Ummul-Nazrah (2017: 58).

Notes. Raven (1964) considered the pantropical Ludwigia octovalvis to be represented by four subspecies, two of which (subsp. octovalvis and subsp. sessiliflora (Micheli) P.H.Raven) occurred in Malesia. However, Raven (1977) retracted this infraspecific classification in favour of one broadly defined and variable species. The synonymy above only includes names relevant to the two former subspecies recognised for Malesia. When Bailey published Jussiaea suffruticosa var. angustifolia and var. villosa he provided a brief description of each variety, but did not cite any potential basionyms for new combinations. Later however, Bailey (1913: 215) did cite Jussiaea angustifolia Lam. and J. villosa Wight \& Arn. as synonyms of his varieties. The first can be taken directly as a basionym, the second provides a direct citation of $J$. villosa Lam. I therefore consider that it was Bailey's intention to make new combinations at varietal rank when he published these names and take them as such based on the Lamarck species names.

\section{LUDWIGIA PERENNIS L.}

Ludwigia perennis L., Sp. Pl. 1 (1753) 119. Jussiaea perennis (L.) Brenan, Kew Bull. 8 (1953) 163. - Type: Herb. Hermann 2: 9, No. 66 (Lectotype BM [BM000621529], designated by Brenan in Turrill \& Milne-Redhead, Fl. Trop. E. Africa, Onagraceae (1953) 13).

Jussiaea suffruticosa L., Sp. Pl. (1753) 388. Ludwigia suffruticosa (L.) M.Gómez, Anales Soc. Esp. Hist. Nat. 23 (1894) 66, nom. illegit., non Walter (1788). - Type: [published illustration] 'Carambu' in Rheede, Hort. Malab. 2 (1679) t. 49 (Lectotype designated here).

Ludwigia oppositifolia L., Syst. Nat., ed. 12, 2 (1767) 125. - Type: Herb. Hermann 2: 9, No. 66 (Lectotype BM [BM000621529], designated here). 
Jussiaea caryophyllaea Lam., Encycl. 3 (1789) 331. - Ludwigia caryophyllaea (Lam.,) Merr. \& F.P.Metcalf, Lingnan Sci. J. 16 (1937) 396. Type: India, Sonnerat s.n. (Lectotype P-LA [IDC 243/7], designated by Raven, Reinwardtia 6 (1964 ['1963']) 367).

Ludwigia parviflora Roxb. Fl. Ind. 1 (1820) 440. 1820. - Isnardia parviflora (Roxb.) Kuntze, Revis. Gen. Pl. 1 (1891) 250. - Type: [unpublished illustration] Icones Roxburghianae 1340 (Lectotype $\mathrm{K}$, designated here).

Ludwigia lythroides Blume, Bijdr. Fl. Ned. Ind. (17) (1826-1827) 1134. - Type: Java, Batavia, Blume 1132 (Lectotype L [L0008663], designated by Raven, Reinwardtia 6 (1964 ['1963']) 367).

Isnardia multiflora Guill. \& Perr., Fl. Senegamb. Tent. (1838) 295. - Ludwigia multiflora (Guill. \& Perr.) Walp., Repert. Bot. Syst. 2 (1843) 75. Type: Sénegambie, Richardtal, 1824, Leprieur s.n. (Lectotype G [G00383323], designated by Brenan in Turrill \& Milne-Redhead, Fl. Trop. E. Africa, Onagraceae (1953) 13; possible Isolectotype P [P00412956]).

Isnardia diffusa Oken, Allg. Naturgesch. 3(3) (1841) 1874. - Type: [published illustration] 'Carambu' in Rheede, Hort. Malab. 2 (1679) t. 49 (Lectotype designated here).

Ludwigia gracilis Miq., Fl. Ned. Ind. 1 (1) (1856) 629. - Type: Sumatra, Padang, Junghuhn s.n. (Lectotype L [L0008662], designated by Raven, Reinwardtia 6 (1964 ['1963']) 367).

Ludwigia nesaeoides H.Perr., Not. Syst. 13 (1947) 141. - Type: Madagascar, Boina, near Marovoay, May 1922, H. Perrier de la Bâthie 17965 (Lectotype P [P00412981], designated at the first step by Raven, Reinwardtia 6 (1964 ['1963']) 368 and at the second step here; Isolectotype $\mathrm{P}$ [P00412982]).

Ludwigia humbertii Robyns \& Lawalrée, Bull. Jard. Bot. État Bruxelles 18 (1947) 291. - Type: Congo, south of Lake Edward, Apr-May 1929, $H$. Humbert 8709 (Lectotype BR [BR000000897374], designated by Raven, Reinwardtia 6 (1964 ['1963']) 368; Isolectotype P [P00412957]).

Description and distribution. Raven (1964: 367), Vu \& Vidal (1973: 27), Khan \& Shamsunnahar (1977: 7), Raven (1977: 103), Kostermans et al. (1987: 374), Thompson (1990: 227), Wagner (1995: 338), Barua (2010: 65), Boufford \& Raven (2014: 605), Ummul-Nazrah (2017: 50).

Notes. Jussiaea suffruticosa L. was, for many years, the accepted name for what is now called Ludwigia octovalvis. In the protologue Linnaeus gave a brief description, 'Jussiaea erecta villosa, floribus tetrapetalis octandris pedunculatis', cited a few references and stated 'Habitat in India'. The only cited element eligible as type material is
Rheede's Carambu, more specifically Rheede's t. 49. This is generally identified as Ludwigia perennis L. and was cited by Linnaeus in the protologue of that name. Ludwigia perrenis is notable for having four stamens, rather than the eight noted in Linnaeus's description of Jussiaea suffruticosa. It has been suggested that Linnaeus really intended to cite Rheede's 'Cattu-carambu' (Rheede, Hort. Malab. 2 (1679) t. 50) (Nicolson et al., 1988), but there is no evidence of Linnaeus correcting himself in this regard in later publications. Fortunately, as Ludwigia suffruticosa Walt. makes the combination in Ludwigia for Jussiaea suffruticosa illegitimate, there are no nomenclaturally disruptive consequences from typifying Jussiaea suffruticosa on t. 49. Given the lack of an alternative under current rules, this is done here.

Linnaeus appears to have changed his mind about the name Ludwigia perennis and used Ludwigia oppositifolia from 1767, citing the former as a synonym of the latter in 1774 . While Ludwigia oppositifolia $\mathrm{L}$. has been referred to as an illegitimate superfluous name it fails to meet the requirements of the code for being superfluous (Art. 52). In the protologue Linnaeus did not cite Ludwigia perennis, or all the elements cited under that name, or use the exact phrase name. Therefore, as it is can clearly be inferred that Linnaeus was basing $L$. oppositifolia on the same elements as $L$. perennis, I here lectotypify it to the same specimen as L. perennis.

\section{LUDWIGIA PERUVIANA (L.) H.Hara}

Ludwigia peruviana (L.) H.Hara, J. Jap. Bot. 28 (1953) 293. - Jussiaea peruviana L., Sp. Pl. (1753) 388. - Jussiaea grandiflora Ruiz \& Pav., Anales Inst. Bot. Cavanilles 14 (1956) 753, nom. illegit., superfl., non Michx. (1803). - Type: [published illustration] "Onagra Laurifolia, flore amplo, pentapetalo" in Feuillée, J. Obs. 2 (1714) t. 9 (Lectotype designated by Raven, Reinwardtia 6 (1964 ['1963’]) 346).

Oenothera hirta L., Syst. Nat. ed. 10, 2 (1759) 998. - Jussiaea hirta (L.) Sw., Obs. Bot. (1791) 142, nom. illegit., non Lam. (1789). - Ludwigia hirta (L.) M.Gómez, Anales Soc. Esp. Hist. Nat. 23 (1894) 66. - Type: [published illustration] 'Oenothera hirsuta' of Plumier in Burman, Pl. Amer. 8 (1758) 167 (Lectotype designated by Ramamoorthy \& Zardini, Monogr. Syst. Bot. Missouri Bot. Gard. 19 (1987) 29).

Jussiaea macrocarpa Kunth, Nov. Gen. Sp. 6 (1823) 102. - Jussiaea peruviana var. macrocarpa (Kunth) Bertoni, Descr. Fis. Econ. Paraguay (1910) 13. - Type: Colombia, Guaduas, Humboldt \& Bonpland 1758 (Lectotype P-Bonpl. [P00679566], designated at the first step by Raven, Reinwardtia 6 (1964 ['1963']) 345), and at the second step here; Isolectotype P [P00135180]). 
Jussiaea mollis Kunth, Nov. Gen. Sp. 6 (1823) 102. - Type: Venezuela, Sucre, Bordones, Humboldt \& Bonpland 414 (Lectotype P-Bonpl. [P00679567], designated by Raven, Reinwardtia 6 (1964 ['1963']) 345).

Jussiaea hirsuta Vell., Fl. Flumin. (1829) 186, nom. illegit., non Miller (1768). - Type: [unpublished illustration] 'Iussieva hirsuta' [original drawing for Vellozo, Fl. Flumin. Icon. 4 (1831) t. 144] (Lectotype Biblioteca Nacional Manuscript Section mss1198653, designated here).

Jussiaea peruviana var. australis Hassl., Repert. Spec. Nov. Regni Veg. 12 (1913) 369. - Type: Paraguay, in regione fluminis Alto Paraná, 1909/1910, Fiebrig 6063 (Lectotype B [B100249031], designated here; isolectotypes G, SI, US).

Jussiaea peruviana var. glaberrima Donn.Sm., Bot. Gaz. 16 (1891) 6. - Type: Guatemala, Depart. Zacatepequez, Dueñas, April 1890, J. Donnell Smith 2130 (Lectotype US [US00123985], designated by Ramamoorthy \& Zardini, Monogr. Syst. Bot. Missouri Bot. Gard. 19 (1987) 30; Isolectotypes GH, K [K000533294], NY).

Jussiaea peruviana f. hirsuta Hassl., Repert. Spec. Nov. Regni Veg. 12 (1913) 269. - Type: Paraguay, in viciniis Caaguazú. Paraguay, Hassler 9167 (Lectotype B [B100249033], designated by Raven, Reinwardtia 6 (1964 ['1963']) 345).

Jussiaea peruviana f. tomentosa Hassl., Repert. Spec. Nov. Regni Veg. 12 (1913) 269. - Type: Paraguay, in altoplanitie et decliviis Sierra de Maracayú, Hassler 5011 (Lectotype B [B100249032], designated here; isolectotypes $\mathrm{G}, \mathrm{GH}, \mathrm{P}, \mathrm{POM})$.

Jussiaea speciosa Ridl., J. Bot. 59 (1921) 259. Type: India, Madras, Vellangiri Hills, Devala, November 1886, Gamble 18361 (Lectotype BM [BM01371212], designated by Raven, Reinwardtia 6 (1964 ['1963']) 345; Isolectotype K [K001129807]).

Jussiaea sprengeri L.H.Bailey, Stand. Cycl. Hort. 3 (1915) 1730. - Type: USA, in cultivation, Johnson s.n. (Lectotype POM, designated by Ramamoorthy \& Zardini, Monogr. Syst. Bot. Missouri Bot. Gard. 19 (1987) 31).

Description and distribution. Raven (1964: 345), Raven (1977: 100), Kostermans et al. (1987: 376), Ramamoorthy \& Zardini (1987: 29), Thompson (1990: 226), Wagner (1995: 334), Barua (2010: 66), Boufford \& Raven (2014: 606), UmmulNazrah (2017: 60).

Notes. Raven (1964) cited the type of Jussiaea mollis Kunth as no. 878. I take this as a typographic error and correct it to 414. Although Ummul-Nazrah (2017) noted the occurrence of Ludwigia peruviana in Sabah and Sarawak, she did not cite any specimens. I have seen collections from Kalimantan (Leeuwenberg 13381, Ambri \& Arifin A.A.455, Arbainsyah AA1861) that confirm the species is naturalized in Borneo.

\section{LUDWIGIA PROSTRATA Roxb.}

Ludwigia prostrata Roxb., Fl. Ind. 1 (1820) 441. Nematopyxis prostrata (Roxb.) Miq., Fl. Ned. Ind. 1(1) (1856) 630. - Isnardia prostrata (Roxb.) Kuntze, Revis. Gen. Pl. 1 (1891) 250. - Jussiaea prostrata (Roxb.) H.Lév., Repert. Spec. Nov. Regni Veg. 8 (1910) 138. - Type: [unpublished illustration] Icones Roxburghianae 1945 (Lectotype K, designated by Vu \& Vidal, Fl. Cambodge, Laos \& Vietnam 14 (1973) 30).

Ludwigia diffusa Buch.-Ham., Trans. Linn. Soc. London 14 (1824) 301. - Type: India, Goyalpara, 2 September 1808, F. Buchanan-Hamilton s.n. [EIC 6336A] (Lectotype K-W [K001123615], designated here).

Ludwigia fruticulosa Blume, Bijdr. Fl. Ned. Ind. (1827) 1133. - Nematopyxis fruticulosa (Blume) Miq., Fl. Ned. Ind. 1 (1) (1856) 630. - Type: Java, Blume s.n. (Lectotype L [L0008664], designated by Raven, Reinwardtia 6 (1964 ['1963']) 374).

Ludwigia leucorhiza Blume, Bijdr. Fl. Ned. Ind. (1827) 1133. - Type: Java, circa Buitenzorg ad flumen Tjeliwong, Blume s.n. (not traced).

Nematopyxis pusilla Miq., Fl. Ned. Ind. 1 (1) (1856) 630. - Type: Java, Horsfield s.n. (Holotype K [K000742277]).

Description and distribution. Raven (1964: 374), Vu \& Vidal (1973: 30), Khan \& Shamsunnahar (1977: 9), Raven (1977: 103), Wagner (1995: 339), Barua (2010: 68), Boufford \& Raven (2014: 607), Ummul-Nazrah (2017: 60).

\section{Species excludenda}

Ludwigia decurrens Walt.

Ramamoorthy \& Zardini (1987: 95) reported a collection of Ludwigia decurrens Walt. from near Laguna in the Philippines (Parchs 6608 (MO)). I have not found any more herbarium material of this species from Malesia and it does not appear to have naturalised in the Philippines (R. Joshi pers. comm., R. Lubigan pers. comm.).

\section{ACKNOWLEDGEMENTS}

The following are thanked for their invaluable assistance in searching for type material: Marc Appelhans, Anand Kumar, Otakar Šida and Jim Solomon. Dr. Nigel Tucker of Biotropica Australia Pty. Ltd. generously supplied information on Ludwigia leptocarpa in Papua New Guinea. David Ples, Dr. Ravindra Joshi and Ross Lubigan very kindly responded to my queries on Philippines Ludwigia during a particularly difficult period in that country. Prof. Marco Pelle- 
grini's assistance with typifying Vellozo names was greatly appreciated. Charlie Jarvis provided guidance with matters relating to the Linnaean names.

\section{REFERENCES}

BAILEY, F. M. 1913. Comprehensive Catalogue of Queensland Plants both Indigenous and Naturalised. A. J. Cumming, Brisbane.

BARUA, I. C. 2020. The genus Ludwigia (Onagraceae) in India. Rheedea 20: 59-70.

BOUFFORD, D. \& RAVEN, P. H. 2014. Onagraceae. Fl. Thail. 11(4): 598-607.

KHAN, M. S. \& SHAMSHUNNAHAR. 1977. Onagraceae. Flora of Bangladesh Fasc. 6: 110. Bangladesh National Herbarium and Bangladesh Agricultural Research Council.

KOSTERMANS, A. J. G. H., WIRJAHARDJA, S. \& DEKKER, R. J. 1987. The weeds: description, ecology and control. In: SOERJANI, M., KOSTERMANS, A. J. G. H. \& TJITROSOEPOMO, G. (Eds.). Weeds of Rice in Indonesia. Balai Pustaka, Jakarta. Pp. 24-565.

LIU, S.-H., HOCH, P. C., DIAZGRANADOS, M., RAVEN, P. H. \& BARBER, J. C. 2017. Multi-locus phylogeny of Ludwigia (Onagraceae): insights on infra-generic relationships and current classification of the genus. Taxon 66: 1112-1127.
NICOLSON, D. H., SURESH, C. R. \& MANILAL, K. S. 1988. An Interpretation of Van Rheede's Hortus Malabaricus. Regnum. Veg. 119: $1-378$.

RAMAMOORTHY, T. P. \& ZARDINI, E. M. 1987. The systematics and evolution of Ludwigia sect. Myrtocarpus sensu lato (Onagraceae). Monogr. Syst. Bot. Missouri Bot. Gard. 19: 1-120.

RAVEN, P. H. (1964 ['1963']). The Old World species of Ludwigia (including Jussiaea), with a synopsis of the genus (Onagraceae). Reinwardtia 6: 327-427.

RAVEN, P. H. 1977. Onagraceae. Fl. Males., ser. I, 8: 98-113.

THOMPSON, J. 1990. Onagraceae. Fl. Austral. 18: 215-243.

TUCKER, N. I. J. 2017. Exotic Plants of the Kikori River Basin. Second Edition. National Quarantine Inspection Authority, Port Moresby.

UMMUL-NAZRAH, A. R. 2017. Onagraceae. Fl. Penins. Malaysia, Ser. II, 6: 49-62.

VU, V. C. \& VIDAL, J. E. 1973. Onagraceae. Fl. Cambodge, Laos, Vietnam 14: 17-39.

WAGNER, W. L. 1995. Onagraceae. Rev. Handb. Fl. Ceylon 9: 332-350.

WAGNER, W. L., HOCH, P. C. \& RAVEN, P. H. 2007. Revised classification of the Onagraceae. Syst. Bot. Monogr. 83: 1-240. 